\title{
Non-Axial View of the Varicella-Zoster Virus Portal Protein Reveals Conserved Crown, Wing and Clip Architecture
}

\author{
Robert J. Visalli ${ }^{\mathrm{a}} \quad$ Alexander J. Howard ${ }^{\mathrm{b}}$

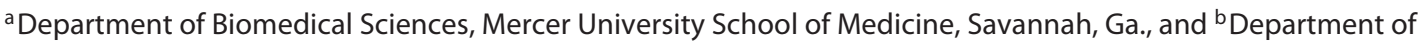 \\ Biology, Indiana University-Purdue University Fort Wayne, Fort Wayne, Ind., USA
}

\section{Key Words}

Encapsidation - Herpesviridae - Portal architecture .

Varicella-zoster virus

\begin{abstract}
Background: Herpesviridae encode a family of protein homologues that function as the 'port of entry' for insertion of the viral DNA into preformed capsids during encapsidation. Methods: Transmission electron microscopy (TEM) of recombinant varicella-zoster virus pORF54 was performed. $\boldsymbol{R e}$ sults: Results suggest that pORF54 forms higher-order structures with itself. Enriched fractions analyzed by TEM revealed non-axial oriented portals with defined central channels and distinguishable crown, wing and clip regions. Conclusion: These morphological features are consistent with those previously reported for other herpesvirus and bacteriophage portal proteins.

๑ 2014 S. Karger AG, Basel
\end{abstract}

\section{Introduction}

Herpesviruses are enveloped, double-stranded DNA viruses that infect both vertebrate and invertebrate animals. Currently, eight different herpesviruses are known to cause disease in humans. All herpesviruses establish latent infections for the lifetime of the host. Human herpesviruses (HHVs) have a distinct tropism for nervous and lymphoid cells, where the body's immune surveillance is limited and the virus can remain undetected. This typically results in cycles of active viral replication (reactivation) and latent periods of infection.

Vaccination has proven valuable for varizella-zoster virus (VZV) and will likely prove useful for the other HHVs in the future. Despite intense efforts by the biomedical research community, there is no effective cure for any established herpesvirus infection. HHVs, including our model organism, VZV, can maintain a latent presence in human populations. As a consequence of latency, therapies which inhibit viral replication cannot effectively eliminate infection. Until a means of resolving latent infection is found, replication inhibitors (i.e. acyclovir) will remain the treatment of choice for suppressing and controlling symptoms of recurrent disease.

Most of the available inhibitors share the same mechanism of action, targeting viral DNA polymerase and interfering with DNA synthesis. These drugs include acyclovir, ganciclovir, penciclovir, brivudin, cidofovir, and foscarnet. They can be categorized into two chemical classes: nucleoside analogues and pyrophosphate analogues [1]. Following phosphorylation of their respective prodrug form, these compounds act as a substrate for viral DNA polymerase, and, once incorporated into the nascent DNA

\section{KARGER}

E-Mail karger@karger.com

www.karger.com/int (c) 2014 S. Karger AG, Basel

0300-5526/14/0572-0121\$39.50/0
Assoc. Prof. Robert J. Visalli, $\mathrm{PhD}$

Mercer University School of Medicine-Savannah Campus

4700 Waters Avenue

Savannah, GA 31404 (USA)

E-Mail Visalli_RJ@mercer.edu 
Table 1. Portal protein homologues of HHVs

a Characteristics

\begin{tabular}{lllllll}
\hline Virus & Subfamily & Portal & $\begin{array}{l}\text { Monomer, } \\
\mathrm{kDa}\end{array}$ & $\begin{array}{l}\text { Length, } \\
\text { AA }\end{array}$ & $\begin{array}{l}\text { Portal complex } \\
\text { mass, MDa }\end{array}$ & Accession \\
\hline HSV-1 & $\alpha$ & pUL6 & 74.1 & 676 & 0.889 & ADM22788 \\
HSV-2 & $\alpha$ & pUL6 & 74.9 & 678 & 0.899 & AEV91344 \\
VZV & $\alpha$ & pORF54 & 86.8 & 769 & 1.042 & ABJ98891 \\
HCMV & $\beta$ & pUL104 & 78.5 & 697 & 0.942 & F5HBR4 \\
HHV-6a & $\beta$ & pU76 & 77.2 & 662 & 0.926 & P52453 \\
HHV-7 & $\beta$ & pU76 & 74.7 & 640 & 0.896 & P52455 \\
EBV & $\gamma$ & pBBRF1 & 68.4 & 613 & 0.821 & AFY97948 \\
HHV-8 & $\gamma$ & pORF43 & 68 & 605 & 0.816 & AAC57125 \\
\hline
\end{tabular}

b Sequence homology

\begin{tabular}{lccccccc}
\hline Virus & HSV-1 & HSV-2 & VZV & HCMV & HHV-6a & HHV-7 & EBV \\
\hline HSV-1 & & & & & & & \\
HSV-2 & $85 / 88$ & & & & & & \\
VZV & $44 / 64$ & $43 / 63$ & & & & \\
HCMV & $24 / 39$ & $25 / 40$ & $23 / 42$ & & & \\
HHV-6a & $23 / 42$ & $24 / 43$ & $24 / 44$ & $36 / 57$ & & \\
HHV-7 & $22 / 42$ & $23 / 41$ & $22 / 43$ & $37 / 60$ & $59 / 77$ & & \\
EBV & $23 / 41$ & $24 / 41$ & $25 / 43$ & $24 / 41$ & $24 / 46$ & $27 / 47$ & $51 / 70$ \\
HHV-8 & $23 / 41$ & $23 / 42$ & $25 / 45$ & $22 / 41$ & $27 / 49$ & $26 / 48$ & 5 \\
\hline
\end{tabular}

$\mathrm{AA}=$ Amino acids. The amount of shading indicates the level of homology between pairs of viruses.

${ }^{1}$ Amino acid identity/similarity.

chain, block strand elongation. Some of these drugs have severe negative side effects, limited viral specificity, and poor bioavailability and/or toxicity profiles. Additionally, since all of these drugs share the same target, development of resistance is possible. Resistant strains of HHVs have been identified for all currently approved drugs [1].

Although therapeutic options are available for certain herpesviruses, shortcomings due to specificity, bioavailability, host toxicity, and drug resistance warrant continued research aimed at identifying and developing novel therapies. Proteins that play a role in herpesviral DNA encapsidation have become promising novel chemotherapeutic targets. Two series of related non-nucleoside compounds, $\mathrm{N}$ - $\alpha$-methylbenzyl- $\mathrm{N}^{\prime}$-aryl thiourea analogues, which inhibit either herpes simplex virus (HSV)-1 $[1,2]$ or VZV $[1,3]$ DNA encapsidation, have been described. In the presence of thiourea compounds, only B capsids were observed in the nuclei of HSV- or VZV-infected cells. Electron microscopy revealed a lack of DNAfilled capsids in the nucleus for HSV-1- or VZV-infected cells treated with their respective thiourea inhibitor $[2,3]$. HSV and VZV mutant viruses resistant to thiourea compounds were found to contain mutations in their putative portal proteins, pUL6 and pORF54, respectively $[2,3]$. In a separate study, the HSV-1 portal protein homologue, pUL6, was shown to be the likely target of the HSV-1-specific thiourea compounds [4]. Previously, pUL6 was shown to localize to a single vertex of the viral capsid and is the likely site of entry for viral genomic DNA during the encapsidation process $[5,6]$. Additionally, HSV-1 UL6 deletion mutants are defective in both DNA cleavage and packaging, which results in large numbers of B capsids in the nuclei of mutant-infected cells. The effect of inhibiting pUL6 or pORF54 function via thiourea compounds is consistent with the genetic evidence provided by studies with HSV-1 deletion mutants $[7,8]$. Thus, a thorough understanding of the interactions between herpesvirus portal proteins and thiourea compounds is of significant interest in the context of developing novel drug treatments for any of the herpesviruses. 
Herpesviruses and dsDNA bacteriophages both utilize a common process to package their viral genomes into empty procapsids during replication. The specific chain of events following assembly of the procapsid and preceding egress of the viral particle from the nucleus (for herpesviruses [9]) or cell (for bacteriophage [10]) is known as DNA encapsidation. The packaging of viral DNA into procapsids is a critical process involving the coordinated interactions of several viral proteins. These include the portal protein, which is located at a single 5 -fold vertex of the procapsid, and a complex of several other proteins known as the terminase. The terminase complex binds and cleaves viral DNA into single genome lengths while interacting directly with the portal protein to translocate viral DNA into the procapsid in an ATP-dependent manner.

The eight portal homologues in human herpesviruses range in molecular mass from $68.0 \mathrm{kDa}$ for HHV-8 to $86.8 \mathrm{kDa}$ for VZV (table 1a). With the exception of VZV, viruses of the same subfamily tend to have portal proteins of similar mass, most notably the $\gamma$-herpesviruses Epstein-Barr virus (EBV; $68.4 \mathrm{kDa})$ and HHV-8 (68.0 kDa). All of the herpesviruses contain a conserved central core that is in part responsible for the amino acid homology observed between viruses of all subfamilies (table 1b; online suppl. fig. S1 describes the highly conserved portal core region found in dsDNA viruses and online suppl. fig. S2 compares the conserved core of herpesvirus family portal proteins via Raptor X; for all online suppl. material, see www.karger.com/doi/10.1159/000360225). For example, VZV pORF54 amino acid similarity ranges from $42 \%$ with human cytomegalovirus (HCMV) to $64 \%$ with HSV-1.

It is reasonable to speculate that pORF54 performs a functional role similar to that of pUL6 and other HHV portal proteins as pORF54 shows $44 \%$ amino acid identity with its HSV-1 homologue (table 1b) [1]. These data, in addition to similar results observed via electron microscopy for inhibitor-treated, infected cells $[2,3]$, are predictive of conserved functions for HSV-1 pUL6, VZV pORF54, and the other HHV portals. The location and arrangement of multimeric pUL6 has been resolved within a unique 5 -fold vertex of HSV-1 B capsids. More extensive studies of bacteriophage portals have produced X-ray crystal structures of dodecameric portal proteins for bacteriophage SPP1 [11, 12]. Based on previous studies of the HSV-1 and HCMV portal proteins $[13,14]$, it is reasonable to assume pORF54 can self-assemble into larger, multimeric structures. Assuming that the VZV portal behaves similarly to other herpesvi-
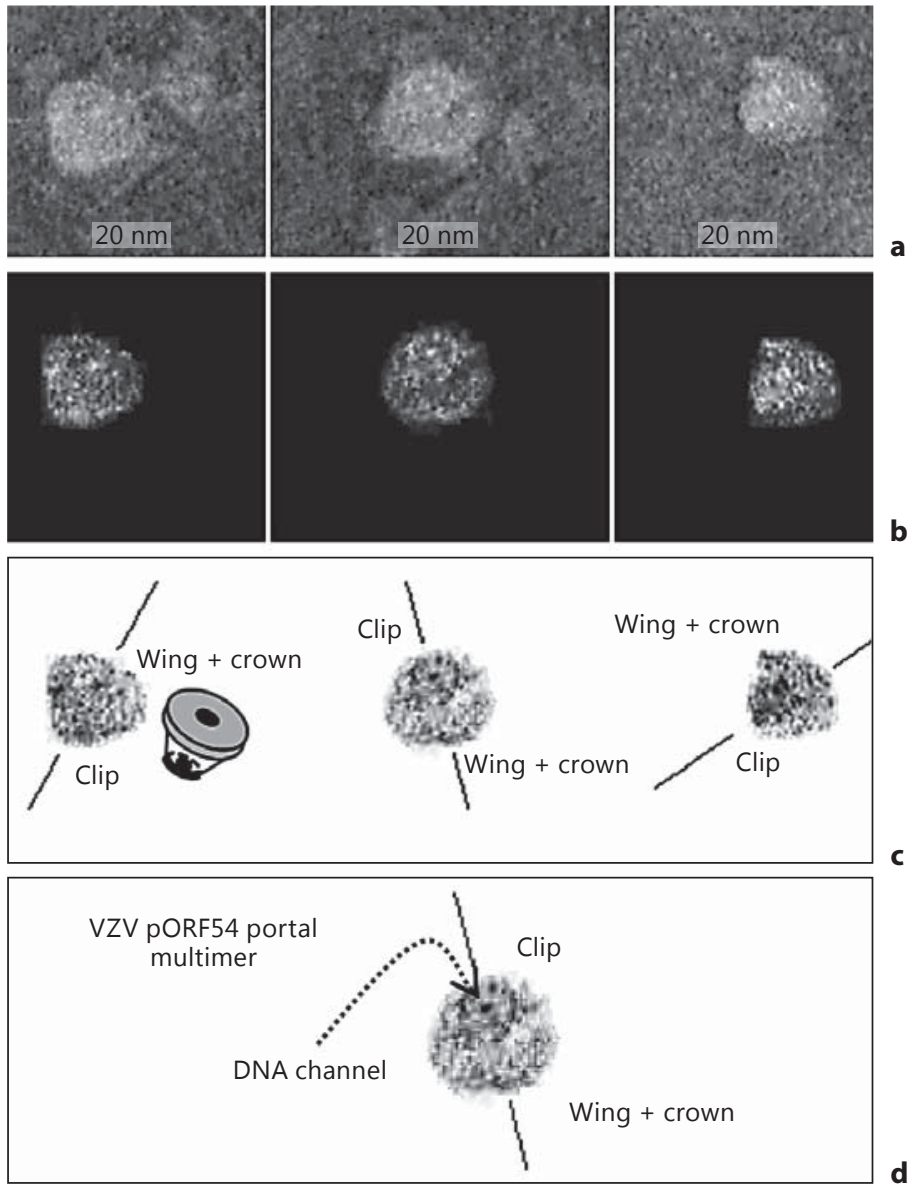

Fig. 1. TEM of purified VZV pORF54 stained with 1\% PTA, glow discharged and imaged at $100 \mathrm{kV}$ using a Philips CM-100 TEM. Individual portals with non-axial perspectives. a Untouched images. The central channel is visible in two of the three (middle and left) portals, while the last presents a more lateral view (right). b Contrast-enhanced view with background noise removed. c Contrast-enhanced negative images with background noise removed. The crown, wing and clip faces are indicated. d DNA channel is visible in the center of the portal multimer.

rus portals, a dodecameric structure would yield a complex of $>1.04 \mathrm{MDa}$ - the largest of the HHV portal proteins (table 1a).

Previously, we performed transmission electron microscopy (TEM) on purified pORF54 expressed in a recombinant baculovirus system. It was the first report of the VZV portal protein [15]. However, we were not able to obtain any non-axial orientations of the portal complex. In this study, samples of the previously isolated pORF54 were diluted in $1.0 \mathrm{M}$ arginine buffer at room temperature and gently sonicated with a probe sonicator. TEM revealed a more even distribution of portals, i.e. less 


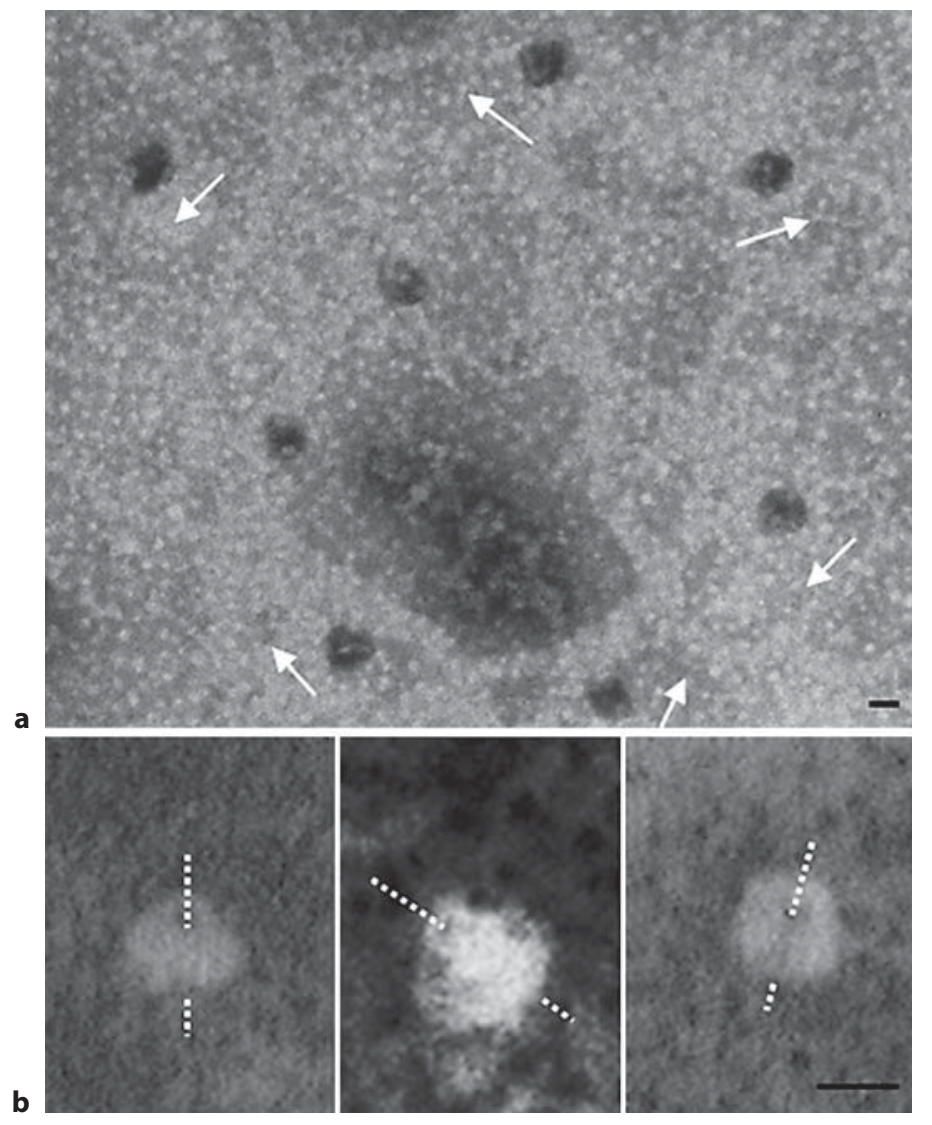

Fig. 2. TEM of VZV portal images stained with $1 \%$ PTA without glow discharge. a Portals were observed in multiple non-axial orientations. The crown/wing versus the clip can be distinguished in most cases. Arrows indicate similar orientation of the portals. b Contrast-enhanced, reverse images of non-glow-discharged portal proteins. The dashed lines indicate the approximate channel path. Bars $=25 \mathrm{~nm}$.

aggregation and elimination of portal 'balls', and showed structural features of the VZV portal not observed previously.

TEM of purified, diluted, and sonicated VZV pORF54 stained with $1 \%$ phosphotungstic acid (PTA) on glowdischarged grids is shown in figure 1.

Individual portal complexes with non-axial perspectives were observed. Three examples of typical portal proteins, with sizes $\sim 25 \mathrm{~nm}$ in diameter, are shown (fig. 1ac). The central channel is visible in two of the three portals (left and center panels), while the last presents a more lateral view (right). The crown, wing and clip faces are indicated. In figure $1 \mathrm{~d}$, a very distinct central channel, presumably the entry point for viral DNA, was observed. The VZV portal is predicted to be larger than any other portal studied to date. This may explain why certain structural features such as the crown/wing and stem are readily visible in the three examples.

In other TEM analysis (fig. 2), samples were stained with $1 \%$ PTA but not glow discharged. Particles were observed with a mushroom-like appearance consistent with the predicted multimeric structure of phage portal proteins for which crystallographic data exist (fig. 2a). Figure $2 \mathrm{~b}$ shows contrast-enhanced images of non-glow-discharged portals. The central channel can be observed in all three cases. The similarity of these images to those observed for the HCMV portal by Holzenburg et al. [13] is striking. These TEM studies represent the most revealing examples to date of intact VZV portal proteins.

A number of unique non-nucleoside $a$-herpesvirus inhibitors have been identified. Novel small molecules that target VZV capsid formation [16] and the HSV helicaseprimase [17-19] are under study. Further investigation of herpesvirus portals and their associated terminase proteins will likely yield new targets for antiviral drug development. The identification of encapsidation-specific antiviral inhibitors for HSV-1, HCMV, and VZV suggests that the encapsidation process is a valid antiviral target for herpesviral chemotherapy [1-3, 20, 21]. It will be interesting to examine portal formation in the presence of inhibitors in order to identify the precise mechanism of action of the thiourea series. Future studies will focus on the effects of inhibitor treatment on the functional and structural characteristics of VZV portal protein.

\section{Acknowledgment}

We would like to thank M.A. Visalli for editorial assistance and D.M. Sherman of the Life Science Microscopy Facility at Purdue University. These studies were supported by National Institutes of Health grant 1 R15 AI062713-02.

References

1 Visalli RJ, van Zeijl M: DNA encapsidation as a target for anti-herpesvirus drug therapy. Antiviral Res 2003;59:73-87.

-2 van Zeijl M, Fairhurst J, Jones TR, Vernon SK, Morin J, LaRocque J, Feld B, O’Hara B, Bloom JD, Johann SV: Novel class of thiourea compounds that inhibit herpes simplex virus type 1 DNA cleavage and encapsidation: resistance maps to the UL6 gene. J Virol 2000;74:90549061.

- 3 Visalli RJ, Fairhurst J, Srinivas S, Hu W, Feld B, DiGrandi M, Curran K, Ross A, Bloom JD, van Zeijl M, et al: Identification of small molecule compounds that selectively inhibit varicella-zoster virus replication. J Virol 2003;77: 2349-2358. 
4 Newcomb WW, Brown JC: Inhibition of herpes simplex virus replication by WAY150138: assembly of capsids depleted of the portal and terminase proteins involved in DNA encapsidation. J Virol 2002;76:1008410088.

$>5$ Cardone G, Winkler DC, Trus BL, Cheng N, Heuser JE, Newcomb WW, Brown JC, Steven AC: Visualization of the herpes simplex virus portal in situ by cryo-electron tomography. Virology 2007;361:426-434.

6 Newcomb WW, Juhas RM, Thomsen DR, Homa FL, Burch AD, Weller SK, Brown JC: The UL6 gene product forms the portal for entry of DNA into the herpes simplex virus capsid. J Virol 2001;75:10923-10932.

7 Lamberti C, Weller SK: The herpes simplex virus type 1 UL6 protein is essential for cleavage and packaging but not for genomic inversion. Virology 1996;226:403-407.

$>8$ Patel AH, Rixon FJ, Cunningham C, Davison AJ: Isolation and characterization of herpes simplex virus type 1 mutants defective in the UL6 gene. Virology 1996;217:111-123.

$>9$ Brown JC, Newcomb WW: Herpesvirus capsid assembly: insights from structural analysis. Curr Opin Virol 2011;1:142-149.
10 Rao VB, Feiss M: The bacteriophage DNA packaging motor. Annu Rev Genet 2008;42: 647-681.

11 Lebedev AA, Krause MH, Isidro AL, Vagin AA, Orlova EV, Turner J, Dodson EJ, Tavares P, Antson AA: Structural framework for DNA translocation via the viral portal protein. EMBO J 2007;26:1984-1994.

12 Olia AS, Prevelige PE Jr, Johnson JE, Cingolani G: Three-dimensional structure of a viral genome-delivery portal vertex. Nat Struct Mol Biol 2011;18:597-603.

13 Holzenburg A, Dittmer A, Bogner E: Assembly of monomeric human cytomegalovirus pUL104 into portal structures. J Gen Virol 2009;90:2381-2385.

14 Rochat RH, Liu X, Murata K, Nagayama K, Rixon FJ, Chiu W: Seeing the portal in herpes simplex virus type $1 \mathrm{~B}$ capsids. J Virol 2011; 85:1871-1874.

15 Howard AJ, Sherman DM, Visalli MA, Burnside DM, Visalli RJ: The varicella-zoster virus ORF54 gene product encodes the capsid portal protein, pORF54. Virus Res 2012;167:102105.
6 Inoue N, Matsushita M, Fukui Y, Yamada S, Tsuda M, Higashi C, Kaneko K, Hasegawa H, Yamaguchi T: Identification of a varicellazoster virus replication inhibitor that blocks capsid assembly by interacting with the floor domain of the major capsid protein. J Virol 2012;86:12198-12207.

17 Vere Hodge RA, Field HJ: Antiviral agents for herpes simplex virus. Adv Pharmacol 2013; 67:1-38.

18 Kleymann G, Fischer R, Betz UA, Hendrix M, Bender W, Schneider U, Handke G, Eckenberg P, Hewlett G, Pevzner V, et al: New helicase-primase inhibitors as drug candidates for the treatment of herpes simplex disease. Nat Med 2002;8:392-398.

19 Chono K, Katsumata K, Kontani T, Kobayashi M, Sudo K, Yokota T, Konno K, Shimizu Y, Suzuki H: ASP2151, a novel helicase-primase inhibitor, possesses antiviral activity against varicella-zoster virus and herpes simplex virus types 1 and 2. J Antimicrob Chemother 2010;65:1733-1741.

20 Biron KK: Antiviral drugs for cytomegalovirus diseases. Antiviral Res 2006;71:154-163.

-21 Bogner E: Human cytomegalovirus terminase as a target for antiviral chemotherapy. Rev Med Virol 2002;12:115-127. 